Revue de droit comparé du travail et de la sécurité sociale

$2 \mid 2020$

La vie personnelle du salarié

\title{
Introduction - La vie privée du salarié dans la jurisprudence comparée
}

Allison Fiorentino

\section{(2) OpenEdition}

1 Journals

Édition électronique

URL : https://journals.openedition.org/rdctss/1049

DOI : $10.4000 /$ rdctss. 1049

ISSN : 2262-9815

Éditeur

Centre de droit comparé du travail et de la sécurité sociale

Édition imprimée

Date de publication : 1 juin 2020

Pagination : 6-13

ISSN : 2117-4350

Référence électronique

Allison Fiorentino, «Introduction - La vie privée du salarié dans la jurisprudence comparée », Revue de droit comparé du travail et de la sécurité sociale [En ligne], 2 | 2020, mis en ligne le 01 novembre 2021, consulté le 11 novembre 2021. URL : http://journals.openedition.org/rdctss/1049 ; DOI : https:// doi.org/10.4000/rdctss. 1049

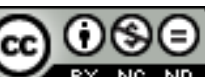

Revue de droit comparé du travail et de la sécurité sociale est mise à disposition selon les termes de la Licence Creative Commons Attribution - Pas d'Utilisation Commerciale - Pas de Modification 4.0 International. 


\title{
ALLison Fiorentino
}

Maître de conférences à I'Université de Rouen, Membre du Centre Universitaire Rouennais d'Etudes Juridiques (CUREJ, EA 4703)

\section{RÉSUMÉ}

Le présent article ouvre le dossier en comparant les jurisprudences françaises, américaines, chinoises et canadiennes sur deux thèmes: les liaisons entre salariés et l'utilisation par ces derniers des réseaux sociaux. Le législateur est parfois intervenu en la matière mais incontestablement c'est le juge qui reste maître du l'application du régime juridique, ne serait-ce qu'en raison de la subjectivité du thème. Qu'est-ce que la vie privée? Dans quelle mesure doit-elle être protégée par rapport aux attentes légitimes d'un employeur ? L'analyse de la jurisprudence met en lumière de grandes divergences suivant les pays. Cependant il est intéressant de constater que certaines solutions sont comparables et reposent parfois sur le bons sens plutôt que sur des constructions jurisprudentielles : c'est le cas des sanctions qui frappent les salariés trop indiscrets qui utilisent les réseaux sociaux.

Mots clés : Réseaux sociaux, liaison, intimité, diffamation, liberté d'expression

\begin{abstract}
This article opens the chronicle by comparing French, American, Chinese and Canadian case law on two themes: affairs between employees and their use of social networks. The legislator has sometimes intervened in this matter, but it is unquestionably the judge who remains in control of the application of the legal system, if only because of the subjectivity of the subject. What is privacy? To what extent must it be protected in relation to an employers legitimate expectations? An analysis of the case law reveals wide divergences between countries. However, it is interesting to note that some solutions are comparable and are sometimes based on common sense rather than on jurisprudential constructions: this is the case of sanctions against overly indiscreet employees who use social networks.
\end{abstract}

Keywords: Social Networks, Liaison, Intimacy, Defamation, Freedom of Expression 


\section{JURISPRUDENCE SOCIALE COMPARÉE}

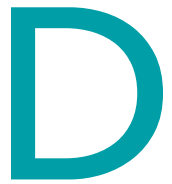

ans quelle mesure la vie privée du salarié peut-elle être protégée? Vie privée et vie professionnelle sont appelées à s'emmêler parfois inextricablement pour finalement s'opposer. En effet, le salarié, comme tout individu, peut revendiquer le droit à une certaine intimité, mais la délimitation de celle-ci implique de déterminer les frontières que l'employeur ne peut franchir. Cela n'est pas chose aisée ne serait-ce que parce que les cas de tensions sont très nombreux. La question de la vie privée du salarié impacte nécessairement les tests que peut faire subir l'employeur : tests de dépistage de drogues, obtention des antécédents de crédit des employés, tests de dépistage du HIV, tests génétiques, la vérification du passé pénal, tests psychologiques... La question de la surveillance du salarié est également cruciale : surveillance de l'utilisation des ordinateurs, téléphones et appareils électroniques pour localiser les salariés, fouille des bureaux et lieux de travail, ou encore usage de dispositifs de vidéosurveillance. Enfin, d'autres situations problématiques pour l'entreprise peuvent être liées à la revendication par le salarié de sa vie privée : liberté vestimentaire, relations affectives avec un autre salarié...

Ces divers exemples mettent inévitablement en exergue la tension existant entre deux ensembles de principes contradictoires. D'une part, il y a le principe de l'inviolabilité du droit à la vie privée du salarié. D'autre part, il y a le droit de l'employeur de jouir de ses biens et d'exercer ses pouvoirs de direction pour protéger ses biens contre les abus potentiels de ses salariés. La légitimité de ces prétentions respectives est indéniable, mais la question de l'équilibre est délicate et requiert toute la délicatesse d'un travail prétorien. En effet, seul le juge est confronté aux situations pragmatiques qui l'amèneront à mettre en balance vie privée et droit de propriété. L'objet du présent dossier est de souligner l'importance de la jurisprudence dans la recherche de cet équilibre.

La multitude des situations dans lesquelles une confrontation entre ces deux principes est inévitable rend difficile l'élaboration d'un panorama exhaustif sur la question de la vie privée du salarié en droit comparé. II semble donc pertinent de débuter l'analyse de ce dossier par deux exemples : la liaison consensuelle entre salariés (jurisprudence française et américaine) (I) et l'usage par les salariés des réseaux sociaux (décisions chinoises et italiennes) (II).

\section{I- LES LIAISONS DANGEREUSES EN DROIT DU TRAVAIL FRANÇAIS ET AMÉRICAIN}

La situation évoquée ici est celle de rapports purement consensuels entre deux salariés. Cela exclut donc les cas de harcèlement, qui constitueraient une faute et justifieraient à ce titre un licenciement. La liaison amoureuse sur le lieu de travail, pour discrète qu'elle soit, n'est pas sans risque pour le salarié. Deux positions prétoriennes antagonistes peuvent être utilisées : celle du juge français et celle de son homologue américain.

En France le salarié bénéficie indubitablement d'un avantage qui s'appuie sur deux textes. D'une part, l'article 9 du Code Civil dispose que « Chacun a droit au respect de sa 
vie privée ", excluant d'emblée l'immixtion d'un employeur dans une relation amoureuse qui ne génère aucun trouble dans l'entreprise. D'autre part, le Code du travail prohibe, à I'article L. 1132-1, toute discrimination directe ou indirecte « en raison de ses mœurs ou de son orientation sexuelle (...) ou de sa situation de famille (...) ». La relation amoureuse n'est pas expressément visée mais cet article pourrait étendre son champ de protection à la vie sentimentale de manière générale.

En $2006^{1}$ la Cour de cassation avait jugé dépourvu de cause réelle et sérieuse le licenciement d'une caissière de supermarché en raison d'une liaison avec un collègue de travail. Sans avoir recours à la notion de discrimination, le juge avait noté que l'employeur ne pouvait se prévaloir d'un trouble suffisant pour porter atteinte à la vie privée de la salariée. Ainsi le soulignait le professeur Radé : «Parce qu'ils relèvent de l'intimité de la vie privée, les sentiments doivent être protégés et demeurer dans la sphère privée $»^{2}$.

Toutefois, cette protection dont bénéficie le salarié n'est pas absolue. Si la liaison impacte négativement l'entreprise, l'employeur est en droit d'intervenir. Tout d'abord, le juge français a élaboré depuis les années 1990 une jurisprudence sur la notion de trouble objectif apporté au fonctionnement de l'entreprise ${ }^{3}$. Cette notion de "trouble objectif » s'évalue au regard de l'activité de l'entreprise et de la nature des fonctions du salarié, de la notoriété de l'acte du salarié ou de ses répercussions sur l'entreprise. Usant de la technique du faisceau d'indices, le juge décide, au cas par cas, si l'action d'un salarié relevant de sa vie privée peut être constitutive d'une cause de licenciement, ce dernier étant alors non-fautif. Cette jurisprudence trouve application en matière de relation amoureuse du salarié.

Ainsi il a été jugé que le licenciement d'un salarié était justifié dès lors que ce dernier avait frappé sa concubine, également salariée ${ }^{4}$. L'altercation avait eu lieu en dehors du lieu de travail mais la police était intervenue dans l'entreprise pour procéder à l'arrestation du concubin violent. Le trouble objectif était caractérisé.

Outre le trouble objectif, une autre situation peut justifier un licenciement, cette fois disciplinaire : lorsque la liaison traduit un manquement de la part de l'un des salariés à ses obligations professionnelles. L'arrêt du 25 septembre $2019^{5}$ en est une illustration. Les faits étaient cocasses. Un supérieur hiérarchique entretient une relation avec une subordonnée et échange avec celle-ci des SMS. Cependant, la romance se gâte lorsque la salariée n'obtient pas les avantages qu'elle souhaite et se plaint à la direction d'un harcèlement sexuel. La Chambre sociale, s'appuyant sur la nature consensuelle de la relation (établie par les SMS), écarte le harcèlement et donc la faute grave du salarié, mais valide néanmoins le licenciement disciplinaire au motif que cette relation lui avait fait " perdre toute autorité et toute crédibilité dans l'exercice de sa fonction de direction [...] incompatible avec ses responsabilités», de sorte que «ces faits se rattachaient à la vie de l'entreprise et pouvaient justifier un licenciement disciplinaire ».

1 Cass. Soc. 21 décembre 2006 n05-41140

2 C. Rade, «Amour et travail : retour sur un drôle de ménage », Dr. soc., 2010, p. 35, spéc., p. 39.

3 P. Waquet, "Le "trouble objectif dans l'entreprise" : une notion à redéfinir ", RDT Dalloz, 2006, p. 304 ; P.-H. Antonmattei, « Le licenciement pour trouble objectif », Dr. Soc., 2012, p. 10.

4 Cass. Soc. 9 juill. 2002, n00-45.068; B. Bossu, "Une altercation avec sa concubine peut justifier le licenciement d'un salarié », RJPF, 2003, $\mathrm{n}^{\circ} 2, \mathrm{p} .9$.

5 Cass. Soc. 25 sept. 2019, n¹7-31.171, Gaz. Pal, 3 déc. 2019, n42, note S. Harir ; Bulletin Trav., 2019, $\mathrm{n}^{\circ} 11$, p. 22, note J. Icard. 


\section{JURISPRUDENCE SOCIALE COMPARÉE}

On constate que la protection de la vie privée du salarié fait obstacle à toute rétorsion de l'employeur (sauf exception). La situation du droit américain est inverse. Le professeur Matthew W. Finkin, grand spécialiste de cette question (et dont nous publions le travail dans cette chronique) avait écrit : "En ce qui concerne la vie privée des employés, les États-Unis sont, juridiquement, un pays sous-développé par rapport à une grande partie de l'Europe $»^{6}$. Si l'époque où Henry Ford inspectait les domiciles des travailleurs est révolue ${ }^{7}$, les employeurs n'ont jamais rencontré un obstacle comparable à l'article 9 du Code civil français car il n'existe aucun équivalent en droit américain.

Aux États-Unis les travailleurs bénéficient de très peu de protections juridiques en matière de vie privée. Peu nombreuses sont les situations où un salarié a le droit, dans le cadre d'une procédure régulière, d'accéder, d'inspecter ou de contester les informations collectées ou détenues par l'employeur. II existe une mosaïque de lois fédérales et d'État qui accordent aux salariés des droits limité ${ }^{8}$. A titre d'exemple, l'Electronic Communications Privacy Act de 1986 (ECPA) est la seule loi fédérale qui offre aux travailleurs des protections en matière de confidentialité des communications. L'ECPA interdit l'interception intentionnelle des communications électroniques?.

Ces exceptions ne constituent nullement un moyen de protection efficace de la vie privée des salariés et fort logiquement il en résulte que l'employeur peut s'immiscer dans la vie intime de ses subordonnés. Il lui est possible d'interdire toute relation amoureuse sur le lieu de travail. Une telle interdiction rencontrerait deux obstacles en France : d'une part, le principe de protection de la vie privée et, d'autre part, le droit du juge français de décider si un motif de licenciement est réel et sérieux. Or, en droit américain, un salarié peut être licencié pour n'importe quel motif dès lors qu'une loi spécifique n'interdit pas ce motif. II s'agit du principe de l'employment at will/10. Cette liberté managériale rend donc possible le licenciement de deux salariés qui auraient entretenu une relation affective même consensuelle. L'un des exemples fréquemment cités est celui de l'affaire opposant UPS à I'un de ses salariés, renvoyé pour ce motif ${ }^{11}$. L'aventure amoureuse entre deux salariés de cette entreprise était restée secrète durant quatre ans et n'avait pu impacter négativement l'entreprise. En outre, elle s'était conclue par un mariage. Elle avait toutefois débuté en violation de l'interdiction générale faite aux salariés d'UPS d'entretenir ce genre de liaison, et

6 M. W. Finkin, "Some further thoughts on the usefulness of comparativism in the law of employee privacy 》, Employee Rts. \& Emp. Pol'y J., 2010, vol. 14, n¹, spéc., p. 11.

7 J.Cunningham Wood et M. C. Wood, Henry Ford: Critical Evaluations in Business and Management, Vol. 1, Taylor \& Francis, 2003, p. 163.

8 M. W. Finkin, Privacy in Employment Law, Bloomberg Law, 5 ème éd., 2018.

9 Cependant, l'ECPA contient des lacunes qui facilitent le contrôle des salariés. Tout d'abord, les employeurs sont autorisés à surveiller les réseaux à des fins commerciales. Cela leur permet d'écouter les appels téléphoniques des salariés ou de consulter leurs e-mails. Les employeurs ne peuvent pas surveiller les appels purement personnels. Toutefois, pour déterminer si un appel est personnel, les employeurs doivent généralement écouter certaines parties de la conversation du salarié. Deuxièmement, un employeur peut intercepter des communications lorsqu'il existe un consentement - réel ou implicite - du salarié. Il y a consentement lorsque l'employeur se contente de donner un préavis de la surveillance.

10 A. Fiorentino, "Le licenciement en droit américain : le principe fondamental de l'employementat-will et sa portée contemporaine ", Revue de la recherche juridique. Droit prospectif , 2012, n¹, p. 463.

11 Ellis v. United Parcel Serv., Inc., 523 F.3d 823 (7th Cir. 2008). 
c'est pour cette seule raison que l'un des salariés avait été licencié. En dépit du recours qu'il intenta, aucune compensation ne lui fut accordée. En effet, le juge statua que l'employeur n'avait enfreint aucun texte. Il en serait allé autrement si le salarié avait pu arguer d'une loi qui interdise les discriminations en raison de la vie personnelle. Or un tel texte n'existe pas aux Etats-Unis et tout au plus quelques Etats interdisent-ils les discriminations en raison du statut marital ${ }^{12}$. Au demeurant, le requérant n'aurait pu en bénéficier puisque ce n'est pas son mariage qui avait causé le licenciement.

\section{II - RÉSEAUX SOCIAUX ET DROIT DU TRAVAIL DANS LES JURISPRUDENCES CHINOISES ET CANADIENNES}

En l'espèce, les oppositions entre jurisprudences sont moins franches ${ }^{13}$. II semble que la notion de vie privée (qu'elle ait été ou non explicitement reconnue au bénéfice des salariés) soit écartée dès lors que le salarié commet l'imprudence de rendre publiques certaines informations.

En Chine, il n'y a guère eu de loi ou de règlement qui concerne spécifiquement les questions de protection de la vie privée des employés sur Internet dans le contexte du travail. Un patchwork de règles et de principes quelque peu pertinents s'est développé de manière fragmentaire, au coup par coup, avec pour résultats des obligations peu importantes pour les employeurs et des lacunes considérables en matière de protection des salariés ${ }^{14}$. La Constitution chinoise ne fait pas spécifiquement référence à un droit à la vie privée. L'article 34 prévoit que la dignité personnelle du citoyen est protégée en tant que droit fondamental. L'article 40 protège le caractère privé de la correspondance des citoyens, ce qui couvrirait sans doute celle sur les médias sociaux. Toutefois, les dispositions constitutionnelles ne sont pas d'application directe et ne peuvent être utilisées dans un procès impliquant deux personnes privées.

Il a fallu attendre la loi sur la responsabilité civile, introduite en 2009, pour que le droit à la vie privée soit reconnu comme l'un des droits dont jouit un individu et dont la violation constitue un délit civil passible de poursuites ${ }^{15}$. Les nouvelles dispositions générales du droit civil de 2017 élargissent encore la protection du droit à la vie privée en l'inscrivant aux côtés d'autres droits de la personne. L'article 110 stipule ce qui suit : « Une personne physique jouit des droits à la vie, à l'inviolabilité et à l'intégrité de la personne, à la santé, au nom, à l'image, à la réputation, à l'honneur, à la vie privée et à l'autonomie maritale, entre autres».

A ce jour, la jurisprudence travailliste ne s'appuie principalement que sur une autre disposition : I'article 39 de la loi sur les contrats de travail, en vertu duquel la faute du salarié doit être grave pour que l'employeur puisse justifier le licenciement immédiat et sans indemnité. Ces circonstances incluent les cas où le salarié a gravement enfreint les règles et procédures établies par l'employeur, a causé un préjudice important à l'employeur

12 II en va ainsi de la Californie (California Fair Employment and Housing Act), de l'Alaska (Alaska Human Rights Law) ou encore du Delaware (Delaware Discrimination in Employment Act).

13 A ce propos, voir le numéro spécial sur la jurisprudence en la matière : Revue de droit comparé du travail et de la sécurité sociale, 2014/2, p. 88.

14 M. Zou, "Social Media and Privacy in the Chinese Workplace: Why One Should Not Friend Their Employer on WeChat », Comparative labor law and policy journal 2018, vol. 39, n², p. 389.

15 Op. cit. 


\section{JURISPRUDENCE SOCIALE COMPARÉE}

en raison d'une négligence grave de ses devoirs ou de la recherche d'avantages privés, a simultanément conclu une relation de travail avec un autre employeur qui affecte sérieusement son emploi actuel, ou fait l'objet d'une enquête pénale.

Sur le fondement de ce seul texte, trois courants jurisprudentiels peuvent être identifiés: - Le premier type de décision est relatif au licenciement du salarié pour avoir mis en ligne certaines informations sur les réseaux sociaux. Ainsi, en $2013^{16}$, un employeur a licencié son salarié pour absence non autorisée du travail pendant plus de huit jours. Lors de l'instance, l'employeur a produit des données publiées par le salarié sur sa page Weibo, le montrant prenant des bains de soleil à une date où l'employeur ne lui avait pas donné d'autorisation d'absence. Le juge a admis la recevabilité de ce moyen de preuve. Une autre catégorie d'affaires est relative aux critiques formulées par les salariés à l'encontre de leurs collègues ou de la direction.

- Dans une décision de 201517, un salarié avait signé un accord avec son employeur selon lequel, en cas de dissolution de son contrat de travail, il recevrait une compensation s'il s'abstenait de faire des commentaires susceptibles de nuire à la réputation de l'employeur. Peu avant de quitter son emploi, le salarié a publié sur son compte Weibo un commentaire indiquant que l'employeur faisait fréquemment des retenues de salaire. L'employeur a prétendu que le commentaire du salarié avait gravement porté atteinte à sa réputation, ce qui justifiait le refus de lui verser une quelconque somme après son licenciement. Le salarié fit valoir l'absence d'intention malveillante, d'autant que cette information était avérée. Le juge donna gain de cause au salarié, non pas en se fondant sur la véracité de l'information donnée, mais sur l'illégalité d'une telle compensation. En effet, il n'appartenait pas aux parties de décider de l'indemnité requise en cas de licenciement, puisque la loi protégeait le droit du salarié à une telle indemnité. Il est regrettable que le juge n'ait pas saisi cette occasion pour clarifier la jurisprudence en la matière, et affirmer - ou infirmer- le droit pour un salarié de communiquer une information exacte sur son entreprise.

- En revanche, et il s'agit du troisième courant prétorien, lorsque les informations sont fausses et procèdent, à l'évidence, d'une intention de nuire, le juge fait légitimement preuve de sévérité envers le salarié qui diffame son employeur. Dans une affaire de $2016^{18}$, un enseignant licencié pour incompétence avait posté des commentaires désobligeants sur l'école dans ses groupes WeChat. Ses commentaires consistaient en des accusations selon lesquelles le plaignant n'offrait pas de qualifications appropriées, délivrait de faux diplômes et s'octroyait une grande partie des salaires des étudiants en stage. Au tribunal, le demandeur fit valoir que les affirmations du défendeur étaient fausses et avaient conduit plusieurs étudiants à retirer leur inscription de l'école, beaucoup d'autres étudiants envisageant des actions similaires. Le tribunal a ordonné au défendeur la publication d'excuses publiques écrites et le paiement des dommages et intérêts.

Par ailleurs, au Canada, même si les solutions prétoriennes varient, elles mettent en évidence l'absence de protection envers le salarié trop imprudent pour rester discret sur certaines informations.

Ainsi au Québec, le salarié a un devoir de loyauté envers l'employeur - en vertu de l'article 2088 du Code civil du Québec- selon lequel il est tenu d'agir loyalement et honnêtement

16 Chen c. Di Nuo Wei Ya International Freight Forwarders (Shanghai) Co., Ltd.

17 Beijing Bonatongcheng Technology Co., Ltd. c. Li Chennan.

18 Xi'an Mou Gong Ye Xuexiao c. Tang. 
dans le cadre de son emploi, et même en dehors du temps de travail lorsqu'il s'agit de la réputation et de la vie privée de l'employeur. Ainsi, l'employeur est-il en droit de s'attendre à ce que le salarié s'abstienne de poster des commentaires nuisibles à la réputation de l'entreprise. En outre, les salariés ont droit à la liberté d'expression en vertu de la Charte des droits et libertés de la personne du Québec ${ }^{19}$. L'équilibre que le juge québécois a établi entre ces deux principes illustre plutôt sa volonté de protéger l'entreprise en relevant aisément une atteinte au devoir de loyauté. Toutefois si le juge admet l'existence d'une faute, la sanction du licenciement est parfois censurée.

Trois exemples illustrent ce propos:

- Un agent administratif du service des ressources humaines de la Ville de Montréal a été licencié pour avoir affiché des commentaires négatifs sur Facebook concernant la décision du directeur d'arrondissement d'euthanasier un pit-bull après qu'il ait attaqué des citoyens. Son licenciement a toutefois été réduit à une suspension de six mois ${ }^{20}$.

- Un agent municipal a été licencié pour avoir publié sur Facebook une vidéo d'une chanson offensante qu'il avait lui-même composée, dont les paroles visaient subtilement le directeur des services techniques et le maire. La vidéo se terminait par un geste dégradant. Son licenciement a été réduit à une suspension de six mois ${ }^{21}$.

- Un commis de librairie a été suspendu pour avoir publié des commentaires diffamatoires, portant atteinte à la réputation de l'employeur, sur son blog accessible via Facebook. Les articles invitaient les lecteurs à déposer des plaintes en ligne contre son employeur et critiquaient les valeurs de l'organisation. Sa suspension de trois jours a été confirmée 22 .

Dans I'Ontario, le juge établit également un équilibre en ayant recours au faisceau d'indices, comme le démontre une décision de 2014²3. Un pompier de Toronto fut licencié pour avoir posté des tweets offensants à partir de son compte personnel Twitter. Pour rendre sa décision, le juge s'est largement concentré sur le contenu sexiste, raciste et homophobe des tweets et sur la façon dont cela se répercutait négativement sur l'employeur. Cette situation a été exacerbée par la nature du rôle du salarié, les pompiers occupant une place de confiance au sein de la société et devant être tenus à une norme de conduite plus élevée.

Outre le contenu des messages postés sur les médias sociaux et la nature du poste du salarié, le juge a mis en évidence un faisceau d'indices prétoriens :

- Quel public était destinataire du message posté ?

- Quelle plateforme a été utilisée?

- Quels étaient les détails de la plateforme (par exemple les paramètres de confidentialité)?

$19 \mathrm{http} / /$ www.cdpdj.qc.ca/fr/formation/situations/Documents/fr/CharteResumeSimplifiee.pdf

20 Montréal (Ville) et Syndicat des fonctionnaires municipaux de Montréal (SCFP-429) (Levasseur), $R e, 2014$ Carswell Que 14102 (T.A. Qué.).

21 (Municipalité) et Syndicat des travailleuses et travailleurs de la municipalité de Weedon (CSN) (2015-03),Re, 2016 CarswellQue 3584 (T.A. Qué.).

22 Librairie Renaud-Bray inc. et Syndicat des employées et employés professionnels-les et de bureau, section locale 574 (SEPB-CTC-FTQ)(Beauregard), Re, 2017 CarswellQue 3294 (T.A. Qué.).

23 Toronto (Clty) v Toronto Professional Fire Fighters Association, Local 3888, 2014 CanLII 76886 (ON LA). 


\section{JURISPRUDENCE SOCIALE COMPARÉE}

- Depuis combien de temps le salarié était-il employé ?

- Quelle a été la réaction du salarié à la découverte des messages (repentant ou non)?

Ces quelques exemples mettent en lumière toute la diversité prétorienne sur la question de la vie privée du salarié. Parfois les solutions s'opposent, parfois elles se rejoignent même si les bases juridiques divergent. Afin de présenter de manière plus complète certains droits, le présent dossier regroupe les travaux de chercheurs provenant d'horizons très différents.

Le droit anglo-saxon sera abordé au travers de :

- l'étude en droit canadien du professeur Urwana Coiquaud consacrée à la question du tatouage du salarié ;

- I'analyse par le professeur Gabrielle Golding de la jurisprudence australienne sur l'utilisation des réseaux sociaux par les salariés ;

- l'article par le professeur Peter Upson relatif au dépistage de drogue sur le lieu de travail en droit néo-zélandais ;

- du panorama élaboré par le professeur Matthew W. Finkin sur la vie privée des salariés aux Etats-Unis.

Le dossier se poursuivra par l'étude d'un pays d'Amérique du Sud, le Mexique, à propos duquel le professeur Gabriela Mendizábal Bermúdez présentera la jurisprudence relative à l'aménagement du temps de travail afin de le rendre compatible avec la vie familiale.

Le Moyen-Orient sera envisagé au travers du travail du professeur Melda Sur qui présentera la vie personnelle du salarié dans la jurisprudence turque.

Enfin le droit européen clôturera ce dossier, puisque seront abordés :

- le droit prétorien allemand sous l'angle de la question du licenciement du salarié pour une activité personnelle qui cause un préjudice ou une gêne à l'entreprise. Ce sont les professeurs Marie-Cécile Escande-Varniol et Gerhard Binkert qui ont réalisé ce travail ;

- la jurisprudence russe à l'aune des standarts internationaux concernant la vie privée du salarié par les professeurs Elena Serebryakova et Elena Sychenko;

- la réforme espagnole de 2019 affectant la conciliation entre le travail et la vie familiale par les professeurs Mercedes López Balaguer et Emma Rodríguez Rodríguez ;

- un rappel de la jurisprudence française sur le respect de la vie personnelle sur le lieu et au temps de travail à travers l'inspection des dossiers informatiques du salarié par Sébastien Ranc. 